\title{
A NEW TECHNIQUE TO OBTAIN CLEAR STATISTICAL PARAMETRIC MAP BY APPLYING ANISOTROPIC DIFFUSION TO FMRI
}

\author{
Hae Yong Kim and Javier Oscar Giacomantone \\ Universidade de São Paulo, Escola Politécnica, Brazil \\ $\{$ hae, javier\}@lps.usp.br
}

\begin{abstract}
This paper presents a new, simple and elegant technique to improve the detection of brain regions with increased neuronal activity in functional magnetic resonance imaging (fMRI). This technique is based on the robust anisotropic diffusion (RAD). A direct application of RAD to fMRI does not work, mainly due to the lack of sharp boundaries between activated and non-activated regions. To overcome this difficulty, we propose to estimate the statistical parametric map (SPM) from the noisy fMRI, compute the diffusion coefficients in the SPM-space, and then perform the diffusion in the structural information-removed fMRI data using the coefficients previously computed. These steps are iterated until the convergence. We have tested the new technique in both simulated and real fMRI, obtaining surprisingly sharp and noiseless SPMs with increased statistical significance. We use Receiver Operating Characteristics (ROC) curves to show that the proposed technique is superior than the conventional correlation method.
\end{abstract}

\section{INTRODUCTION}

The purpose of Functional Magnetic Resonance Imaging (fMRI) is to map areas of increased neuronal activity of the human brain. fMRI has been applied to investigate a variety of neuronal processes from activities in the primary sensory and motor cortices to cognitive functions such as perception or learning. The hemoglobin in the blood is a natural contrast agent, because it has different magnetic properties depending on its state of oxygenation. These differences affect the voxel intensity in the magnetic resonance image. In a typical fMRI experiment, baseline images are scanned periodically while the subject is at rest (or in other baseline condition) and activation images are acquired when the subject is performing a specific task or receiving a stimulus. A classical method for identifying activated brain regions from noisy fMRI 4-D image is the correlation analysis [1]. A correlation coefficient measures the degree of matching

This work was supported in part by FAPESP under grants 03/033693 and 03/13752-9, and by CNPq under grants 305065/2003-3 and $475155 / 2004-1$ between the time series of a particular voxel and the expected activation of the experiment design. For each correlation coefficient, a t-statistics is computed and spatially disposed, forming a Statistical Parametric Map (SPM). The classification of voxels as active or non-active is performed by thresholding the SPM at a particular significance level, without taking into account any spatial relationship between voxels.

To include the spatial relationship and improve the detection of active voxels, we proposed a novel technique called RADSPM. It is based on Robust Anisotropic Diffusion (RAD) and classic correlation coefficient analysis. A direct application of RAD to fMRI data does not work, mainly due to the lack of sharp boundaries between activated and non-activated regions. RAD can be directly applied to SPM, but it fails to substantially improve the quality of SPM because it does not take into account the originating fMRI data. To overcome this difficulty, we use the gradient magnitudes estimated in the SPM-space as the arguments of an "edge-stopping" function to compute the diffusion coefficients. The diffusion is then performed in the mean-corrected fMRI data, using the coefficients preciously computed. These steps are iterated until the convergence. We have tested the new technique in both simulated and real fMRI, obtaining surprisingly sharp and noiseless SPMs with increased statistical significance. We use ROC curves to show that the proposed technique is superior than the conventional correlation method.

\section{ROBUST ANISOTROPIC DIFFUSION}

Perona and Malik [2] defined the anisotropic diffusion as

$$
\frac{\partial I(x, y, t)}{\partial t}=\operatorname{div}[g(\|\nabla I(x, y, t)\|) \nabla I(x, y, t)],
$$

using the original image $I(x, y, 0): \mathbb{R}^{2} \rightarrow \mathbb{R}^{+}$as the initial condition, where $t$ is an artificial time parameter and $g$ is an "edge-stopping" function. The right choice of $g$ can greatly affect the extent to which discontinuities are preserved. Perona and Malik suggested two possible edge-stopping functions in their paper [2]. Black et al. [3] used the robust es- 
timation theory to choose a better edge-stopping function, called Tukey's biweight:

$$
g(x)= \begin{cases}{\left[1-\frac{x^{2}}{5 \sigma^{2}}\right]^{2},} & \frac{x^{2}}{5} \leq \sigma^{2} \\ 0, & \text { otherwise }\end{cases}
$$

The function $g$ above is the dilated and scaled version of the original Tukey's function, where $g(0)=1$ and the local maxima of its "influence function" $\psi(x)=x g(x)$ is situated at $x=\sigma$. The diffusion that uses the Tukey's function is called robust anisotropic diffusion (RAD) and this is the edge-stopping function adopted in this paper.

Perona and Malik [2] discretized spatio-temporally their anisotropic diffusion equation (1) as:

$$
I(s, t+1)=I(s, t)+\frac{\lambda}{\left|\eta_{s}\right|} \sum_{p \in \eta_{s}} g\left(\left|\nabla I_{s, p}(t)\right|\right) \nabla I_{s, p}(t)
$$

where $I(s, t)$ is a discretely sampled image, $s$ denotes the pixel position in a discrete 2-D or 3-D grid, $t \geq 0$ now denotes discrete time steps, the constant $\lambda$ determines the rate of diffusion (usually, $\lambda=1$ ), and $\eta_{s}$ represents the set of spatial neighbors of pixel $s$. For 2-D images, usually four neighbors are considered: north, south, west and east, except at the image boundaries. For 3-D images, six voxels are usually considered: the above-mentioned four plus "up" and "down" voxels. The gradient magnitude of a voxel in a particular direction at iteration $t$ is approximated by:

$$
\nabla I_{s, p}(t)=I(p, t)-I(s, t), p \in \eta_{s} .
$$

\section{CORRELATION ANALYSIS}

Correlation analysis is a simple method widely used to detect active voxels in fMRI images [1]. Each voxel $s$ has an associated time series $X_{s}=\left\{x_{1}, x_{2}, \ldots x_{N}\right\}$, where $N$ is the number of volumes of the 4-D functional image. $Y=\left\{y_{1}, y_{2}, \ldots, y_{N}\right\}$ is the reference time series, expected to represent the oxygenation changes in the blood due to the experiment stimulation. The reference time series can be a simple square waveform representing the stimulation protocol, a delayed square waveform, or the convolution of a square waveform with the Hemodynamic Response Function (HRF) of the brain [4]. The sample correlation coefficient

$$
\rho_{s}=\frac{\sum\left(x_{i}-\bar{x}\right)\left(y_{i}-\bar{y}\right)}{\sqrt{\sum\left(x_{i}-\bar{x}\right)^{2} \sum\left(y_{i}-\bar{y}\right)^{2}}}
$$

describes the matching between the observation and the expectation. In order to classify the voxel $s$ as active or nonactive, we transform the correlation coefficient $\rho_{s}$ into the random variable $\tau_{s}$ that follows Student's t-distribution with $N-2$ degrees of freedom:

$$
\tau_{s}=\frac{\rho_{s} \sqrt{N-2}}{\sqrt{1-\rho_{s}^{2}}}
$$

The image obtained by spatially disposing $\tau_{s}$, for every voxel $s$, is called statistical parametric map $\operatorname{SPM}(\tau)$.

\section{THE PROPOSED METHOD}

SPM does not take into account any spatial relationship between voxels. Some papers have proposed to use the anisotropic diffusion to exploit the spatial correlation between activated voxels.

Neoh and Sapiro [5] have applied the anisotropic diffusion directly to the SPM. However, it cannot substantially improve the quality of SPM because it does not take into account the originating fMRI data.

Solé et al. [6] have proposed another technique named anisotropic averaging. It computes an initial set of clearly activated voxels. This set is then used to construct a complex "similarity measure" to compute the averaging coefficients. Even though Sole et al. try to explain the definition of their measure with intuitive arguments, we are impelled to ask if there are no simpler and more natural ways to define the similarity measure. Moreover, Solé et al.'s algorithm iteratively classifies the voxels into two sets: activated and non-activated voxels. Consequently, it is adequate to make binary decisions, but it may not be adequate to get a multi-leveled SPM (many studies actually care about the level of activation and not just the binary decision of activated or not). We propose a new and different technique, directly related to the RAD, that we have named RADSPM ${ }^{1}$. Our method is simple, elegant and has yielded surprisingly clear SPMs when applied to both simulated and real fMRI data. RADSPM increases substantially the statistical significance of activated regions, what makes it possible to decide with more confidence if a certain brain region is activated or not.

Let $I^{\prime}$ be an fMRI data. First of all, the mean value is removed from $I^{\prime}$, yielding the mean-removed fMRI $I$ :

$$
I=I^{\prime}-\overline{I^{\prime}}
$$

This pre-processing is very important, because structural and functional regions of the brain do not necessarily match. No structural information should be diffused, but only the activation information. Note that the activation information is not affected at all by the mean-correction.

Let us denote the fMRI data at iteration $t \geq 0$ of the diffusion process as $I(s, n, t)$, where $I(s, n, 0)$ is the initial mean-corrected fMRI at spatial voxel position $s$ and volume $n$. RADSPM is described below:

$$
\text { 1. Let } t \leftarrow 0 \text {. }
$$

${ }^{1} \mathrm{~A}$ earlier version of this technique was published in a local conference [7]. 
2. Calculate the $\operatorname{SPM}(\tau) T$, using equations 5 and 6 . Let us denote the value of the $\operatorname{SPM}(\tau)$ at voxel $s$ and iteration $t$ as $T(s, t)$.

3. Compute the diffusion coefficients. The diffusion coefficient between a voxel $s$ and its neighboring voxel $p$ at instant $t$ is:

$g\left(\left|\nabla T_{s, p}(t)\right|\right)$, where $\nabla T_{s, p}(t)=T(p, t)-T(s, t)$.

4. Use these coefficients to perform the diffusion in $I(s, n, t)$, yielding the diffused fMRI $I(s, n, t+1)$ at iteration $t+1$ :

$$
\begin{aligned}
& I(s, n, t+1) \leftarrow I(s, n, t)+ \\
& +\frac{\lambda}{\left|\eta_{s}\right|} \sum_{p \in \eta_{s}} g\left(\left|\nabla T_{s, p}(t)\right|\right) \nabla I_{s, p}(t),
\end{aligned}
$$

where $\nabla I_{s, p}(n, t)=I(p, n, t)-I(s, n, t)$.

5. Let $t \leftarrow t+1$ and repeat steps 2 to 5 some predefined number of times.

\section{EXPERIMENTAL RESULTS}

\subsection{Simulated fMRI}

In order to test our technique, we generated a simple artificial 4-D fMRI, with $10 \times 10 \times 3$ voxels per volume and 84 volumes. Voxels values were 16000 corrupted by zeromean Gaussian noise with standard deviation $\sigma=4000$. Active voxels had their values increased by 1500 . The fMRI had alternating blocks of 6 non-active and 6 active volumes, beginning with non-active volumes. Activated volumes had a $6 \times 6$ activated area in the center of the volume, with two non-activated holes with 4 voxels each. Figure 1 depicts one activated volume of the phantom, the $\operatorname{SPM}(\tau)$ generated by the correlation method and the $\operatorname{SPM}(\tau)$ generated by RADSPM. Clearly, RADSPM has produced an $\operatorname{SPM}(\tau)$ with better quality than the correlation.

We will make use of the well-known Receiver Operating Characteristics (ROC) analysis $[8,9,10]$ to compare the correlation and RADSPM techniques. Let TP, FN, FP and TN be respectively the number of true positives, false negatives, false positives and true negatives obtained by comparing the ideal classification (gold standard) and the image obtained by applying a threshold to the $\operatorname{SPM}(\tau)$. Then, the True Positive Fraction (TPF) and the False Positive Fraction (FPF) are defined as:

$$
\mathrm{TPF}=\frac{\mathrm{TP}}{\mathrm{TP}+\mathrm{FN}}, \quad \mathrm{FPF}=\frac{\mathrm{FP}}{\mathrm{FP}+\mathrm{TN}}
$$

Fig. 2 depicts RADSPM's and correlation's ROC curves. Each point of a ROC curve is obtained by solving equation

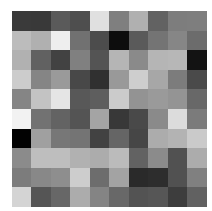

(a) Slice 1

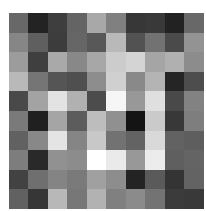

(d) Slice 1

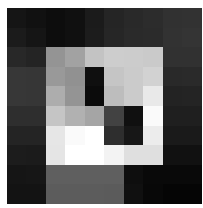

(g) Slice 1

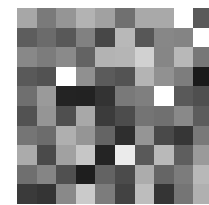

(b) Slice 2

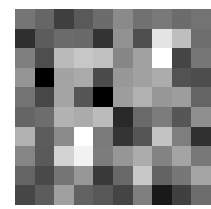

(e) Slice 2

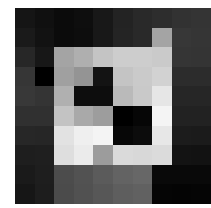

(h) Slice 2

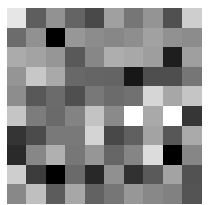

(c) Slice 3

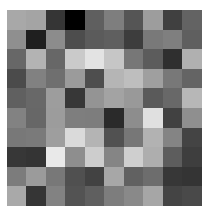

(f) Slice 3

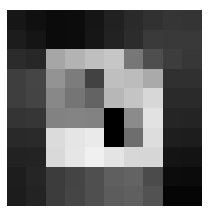

(i) Slice 3
Fig. 1. 1st row: Simulated fMRI. 2nd row: $\operatorname{SPM}(\tau)$ produced by correlation method. 3rd row: $\operatorname{SPM}(\tau)$ produced by $\operatorname{RADSPM}(\sigma=2, t=10)$.

\begin{tabular}{|c|c|c|c|c|c|}
\hline Method & Area & $d_{\text {oop }}$ & TPF $_{\text {oop }}$ & FPF $_{\text {oop }}$ & $p_{\text {oop }}$ \\
\hline Correlation & 0.88 & 0.44 & 0.85 & 0.23 & 0.2 \\
RADSPM & 0.99 & 0.66 & 0.99 & 0.06 & 0.00001 \\
\hline
\end{tabular}

Table 1. Performance metrics.

(8) for a specific threshold value. Table 1 presents some performance metrics of the two ROC curves, all of them demonstrating the superiority of the RADSPM: (1) The area under RADSPM's curve is larger than those of correlation's; (2) The distance $d_{o o p}$ from the principal diagonal to the optimal operating point (OOP) is longer in RADSPM's curve than in correlation's (OOP is the point of the curve most distant from the principal diagonal); (3) At the OOP, RADSPM shows larger TPF, lower FPF and lower observed statistical significance level $p_{\text {oop }}$ than the correlation method.

\subsection{Real fMRI}

We have also tested the correlation and RADSPM techniques with a real fMRI data obtained from a visual block design experiment. Whole-brain BOLD/EPI fMRI data were acquired on a 1.5T Philips Eclipse system. The condition for successive blocks alternated between rest and visual stimulation, starting with rest. Visual stimulation was given by flickering red LED light with frequency of $2 \mathrm{~Hz}$. The volumes were realigned and normalised, resulting fMRI data with $79 \times 95 \times 68$ voxels per volume and 55 volumes. A spin echo T1-weighted image was also obtained to be used 


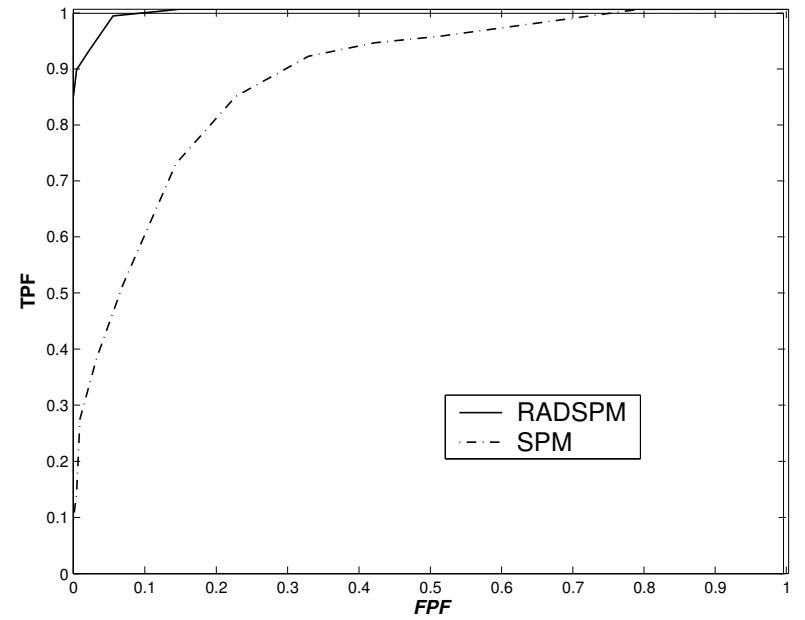

Fig. 2. ROC curves

as the structural image. Figure 3 depicts the SPMs overlaid on the structural MRI. The first row depicts the slices obtained with the correlation and the second row with the RADSPM. Clearly, RADSPM-generated $\operatorname{SPM}(\tau)$ has activated regions with substantially increased statistical significance, what makes it possible to decide with more confidence if a certain brain region is activated or not.

\section{CONCLUSIONS}

In this paper, we have presented a new technique named RADSPM to obtain clear SPMs from noisy fMRI. It is directly inspired by the robust anisotropic diffusion. Experimental results, using both the simulated and real fMRI data, have shown that the proposed method generates surprisingly sharp and noiseless SPMs.

\section{REFERENCES}

[1] Peter A. Bandettini, A. Jesmanoswicz, Eric C. Wong, and James S.Hyde, "Processing strategies for time-course data sets in functional mri of the human brain," Magnetic Resonance in Medicine, vol. 30, pp. 161-173, 1993.

[2] P. Perona and J. Malik, "Scale space and edge detection using anisotropic diffusion," IEEE. Transaction on Pattern Analysis and Machine Intelligence, vol. 12, no. 7, pp. 629-639, 1990.

[3] M. J. Black, G. Sapiro, D. H. Marimont, and D. Hegger, "Robust anisotropic diffusion," IEEE Transaction on Image Processing, vol. 7, no. 3, pp. 421-432, 1998.

[4] Gossl C., Auer D. P., and Fahrmeir L., "Hemodynamic response function in bold fmri," Neurolmage, vol. 14, pp. 140148,2001

[5] Hong Shan Neoh and Guillermo Shapiro, "Using anisotropic diffusion of probability maps for activity detection in blockdesign functional mri," in Proceedings of the IEEE Inter-

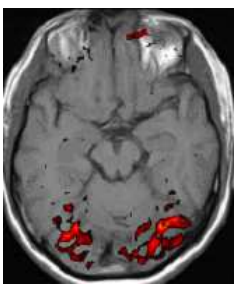

(a) slice 35

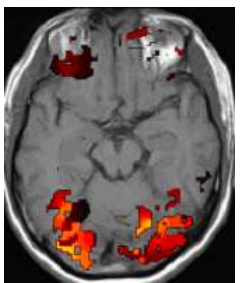

(d) slice $35, \sigma=1.5$

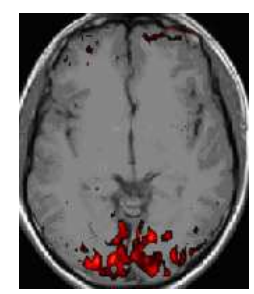

(b) slice 50

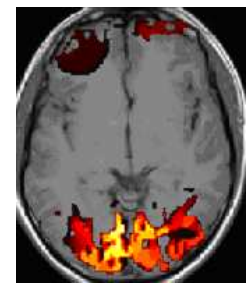

(e) slice $50, \sigma=1.5$

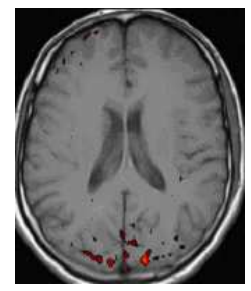

(c) slice 75

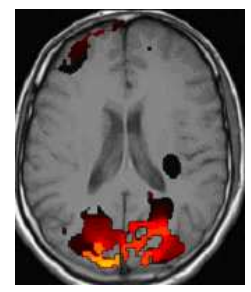

(f) slice $75, \sigma=1.5$
Fig. 3. Real fMRI processed by correlation and RADSPM techniques. 1st row: $\operatorname{SPM}(\tau)$ obtained by correlation (overlaid on the structural MRI). 2nd row: $\operatorname{SPM}(\tau)$ obtained by $\operatorname{RADSPM}(\sigma=1.5, t=90)$.

national Conference on Image Processing, 2000, vol. 1, pp. 621-624.

[6] A. F. Solé, S. C. Ngan, G. Sapiro, X. P. Hu, and A. López, "Anisotropic 2-d and 3-d averaging of fmri signals," IEEE Transaction on Medical Imaging, vol. 20, no. 2, pp. 86-93, 2001.

[7] H. Y. Kim and Z. H. Cho, "Robustsing Anisotropic Diffusion to Produce Clear Statistical Parametric Maps from fMRI," in Proceedings of Sibgrapi - Brazilian Symp. on Comp. Graph. and Image Proc., 2002, pp. 11-17.

[8] R. T. Constable and Skudlarski P., "An ROC approach for evaluating functional brain MR image analysis," Magnetic Resonance in Medicine, vol. 34, no. 1, pp. 57-64, 1995.

[9] J. A Sorenson and X. Wang, "ROC Method for Evaluation of fMRI techniques," Magnetic Resonance in Medicine, vol. 36, pp. 737-744, 1996.

[10] Skudlarski Powel, Constable Todd R., and Gore John C., "ROC Analisis of Statistical Methods Used in Funcional MRI: Individual subjects," Neuroimage, vol. 9, pp. 311-329, 1999. 